\title{
Preterm Birth in the United States: A Sociocultural Approach
}

\author{
by Janet M. Bronstein \\ Dordrecht: Springer International Publishing, 2016 \\ ISBN 978-3-319-32713-6 \\ Hardcover \$179, 337 pp.
}

\section{Reviewed by Savannah Larimore \\ University of Washington}

Despite my initial assumptions, Preterm Birth in the United States: A Sociocultural Approach does not read like a conventional textbook, but rather presents the body of knowledge on pre-term birth with a surprising depth of critical engagement. The author, Janet M. Bronstein, holds a Ph.D. in Applied Medical Anthropology and several joint appointments across the departments of Health Policy, Sociology, and Political Science at the University of Alabama at Birmingham. Bronstein also teaches courses in public health ethics, conducts research on low-income populations and the medical safety net, and serves as an evaluator for family planning and maternity services demonstration programs within Medicaid agencies in Alabama and Arkansas. The diversity and breadth of Bronstein's training and experience allows the author to present comprehensive and nuanced cultural and historical views of preterm birth as well as critical analyses of how this health outcome is defined in academic settings and treated in clinical ones. Bronstein's review and analysis of this topic is both timely and overdue, as the US has consistently fallen well behind peer nations in birth outcomes.

The text is organized by six "dimensions of preterm birth": clinical, population, cultural, political, health care, and ethical. Bronstein argues that these dimensions do not exist in isolation but instead overlap to create one overarching view of preterm birth in the US, and explicitly notes the places that themes connect. Each chapter is additionally organized into distinct subsections or sub-dimensions, and concludes with a comparison of the manifestation of each chapter's dimension in US society with three comparable societies: Canada, Britain, and Western Europe. As the title of the book implies, in each chapter Bronstein shifts the focus from biological, proximate causes of preterm birth towards the distal, cultural, and social causes of this health outcome. This does not mean that Bronstein shies away from discussions of biological causes; when necessary, the author makes a concerted effort to talk about preterm birth as an outcome separate from other birth outcomes — namely, infant mortality and low birth weight — rather than conflating these outcomes and assuming they stem from one singular, common cause, as other texts often do.

Given Bronstein's training, it is no surprise that I found the third chapter, which focuses on the cultural dimension of preterm birth, to be the most compelling. In this chapter, Bronstein applies her insights as a medical anthropologist to the cultural frames and social processes that have shaped historical and contemporary views on motherhood, pregnancy, fetal viability, and preterm birth among other "poor" birth outcomes, namely, infant mortality and low birthweight. For this chapter, Bronstein presents an original analysis of articles from major US newspapers and identi- 
fies three primary narratives with several shared themes: "the hero's journey," "the power of technology," and "expressions of compassion." Bronstein notes that media representations and the narratives present in those representations often demonstrate the ways that a society defines and evaluates social problems. Based on this analysis and other points made in the chapter, it is clear that cultural narratives of preterm birth in the US focus on the use of technology to deliver, and aid in the survival of, infants born at very early gestational ages. Bronstein also notes that preterm birth is one of the few cases in US society where compassion is favoured over rational, calculated decision making, and media representations reflect this by appealing to emotion even when, for various reasons, medical intervention may not appear to be the best course of action.

Despite many strengths of the text, there are several shortcomings throughout. Most importantly, I expected a more intersectional and comprehensive overview of racial disparities in the US and the comparison societies that Bronstein highlights. While I understand that racial disparities are not the sole focus of this book, in areas where racial and ethnic disparities in preterm birth or other birth outcomes are mentioned, Bronstein reinforces an incredibly common problem in this subfield: focusing on the Black-White gap in birth outcomes while minimizing or ignoring other gaps between Whites and racial minorities or across racial and ethnic minority groups. While Bronstein does highlight the Hispanic/Latinx epidemiological paradox, there is little to no mention of birth outcomes and disparities for Asians, Native Hawaiians or Pacific Islanders, and Native Americans or Alaska Natives. Similarly, while I commend Bronstein for explicitly naming the role that slavery and anti-black oppression plays in maintaining and reproducing the Black-White gap in birth outcomes, I found a lack of the same treatment of other forms of racism specific to other groups (e.g., Japanese internment, the Native American genocide, and so on). The same can be said for analyses of racism in the comparison countries which have histories of colonization and racial oppression that, while distinct from that of the US, are still present. For example, Bronstein fails to highlight the similarities in oppression of indigenous populations in the US and Canada, which would have been a worthwhile comparison to make, given the stark difference in health care systems between the two bordering nations.

That said, as a young scholar interested in racial disparities in low birth weight in the US, but not necessarily preterm birth, I consider this book indispensable. I would recommend this book to researchers interested in birth outcomes and women's health in the US, as well as professors teaching upper-division undergraduate and graduate courses in similar topics. Additionally, while the text is written for an academic audience, I find Bronstein's prose to be approachable for many non-academic audiences, and the book's structure makes identifying sub-sections of interest for quick reading possible. Overall, this book was a pleasure to read and I look forward to referencing it many more times in the future. 